\title{
Resistless electron beam lithography process for the fabrication of sub-50 $\mathrm{nm}$ silicide structures
}

\author{
D. Drouin ${ }^{\mathrm{a})}$ \\ Département de Génie Mécanique, Groupe de Recherche sur les Matériaux pour Composants et \\ Microstructures, Université de Sherbrooke, Sherbrooke, Québec J1K 2R1, Canada \\ J. Beauvais, E. Lavallée, S. Michel, and J. Mouine \\ Département de Génie Électrique et de Génie Informatique, Groupe de Recherche sur les Matériaux pour \\ Composants et Microstructures, Université de Sherbrooke, Sherbrooke, Québec J1K 2R1, Canada \\ R. Gauvin \\ Département de Génie Mécanique, Université de Sherbrooke, Sherbrooke, Québec J1K 2R1, Canada
}

(Received 27 May 1997; accepted 21 July 1997)

\begin{abstract}
We report on a study of the fabrication of submicron silicide structures with a resistless lithography technique. Several different metals can be used as a basis for producing silicide using this method; in this work, results will be discussed for both platinum and nickel silicide. The feasibility of producing nanostructures using polycrystalline silicon as a base growth layer for metal-oxidesemiconductor, and other device applications have also been demonstrated. Threshold doses for this method for submicron lines $(<50 \mathrm{~nm})$ and square areas were obtained in order to establish a framework for the fabrication of more complex devices. Preliminary electrical measurements were carried out which indicate that the resistivity of the silicide is $45 \mu \Omega \mathrm{cm}$, and that the barrier height of the silicide/(high resistivity silicon) interface is $0.56 \mathrm{eV}$. (c) 1997 American Vacuum Society. [S0734-211X(97)01506-0]
\end{abstract}

\section{INTRODUCTION}

The continual reduction in size of microelectronic circuits and devices pushes the limits of current lithography processes. Several new techniques are presently under investigation, ${ }^{1,2}$ particularly for the fabrication of ultrasmall devices, in the sub-100 $\mathrm{nm}$ size domain. One of the main focus points of these activities is the development of a resistless lithography process based on the use of an electron beam (EB). Several disadvantages are associated with the use of conventional resists in standard EB lithography. These include proximity effects and resolution limits imposed by the size of the resist molecule. Therefore, several research groups have proposed methods by which chemical reactions can be activated by an EB, allowing for extremely high resolution often without any proximity effects. ${ }^{3,4}$ However the drawback is often the requirement that the EB exposure must be carried out in a reactive atmosphere in the sample chamber. ${ }^{5}$ Recently, our group has proposed a method for a resistless EB lithography without the use of reactive gases. ${ }^{6}$ The EB is used to provide local heating of a thin metal film on a silicon substrate, thus generating silicide structures in the exposed areas. This method does not appear to suffer from the usual problems associated with resist based processes. Furthermore, the type of metal thin film that can be used includes all the metals that can form silicides at reasonably low temperatures. In this article, we will discuss the formation of both platinum and nickel silicide structures using this lithography process. In addition to metalsemiconductor structures (MESs), the technique has been extended to the fabrication of silicide structures over oxide

${ }^{a)}$ Electronic mail: drouin@aramis.gme.usherb.ca layers, using a thin layer of polycrystalline silicon covered with a metal thin film in order to produce the silicide layers. Thus, several types of structures were produced that can be used in the fabrication of metal-oxide-semiconductor (MOS) devices. The results presented here will include a study of threshold exposure doses required for the fabrication of both submicron lines and micrometer sized squares, and a comparison of the thresholds measured for platinum and nickel silicide structures. The effects of the beam energy will also be presented. Electrical measurements will also be discussed for both the MES and MOS structures fabricated using this lithography technique.

\section{DESCRIPTION OF THE METHOD}

Silicide structures are widely used in the microelectronics industry, due to the exceptionally good interface with silicon. Platinum silicide formed on $n$-type silicon produces a Schottky contact with a high barrier height $\left(\phi_{b}>0.8 \mathrm{eV}\right)^{7}$ and is used, e.g., in bipolar integrated circuits. ${ }^{8}$ Silicide formed on $p$-type silicon generates a low barrier $\left(\phi_{b}<0.25\right.$ $\mathrm{eV})^{7}$ that can be used to fabricate infrared detectors. ${ }^{9}$ These contacts are also widely used in MOS technology for low resistivity ohmic contacts. ${ }^{10}$ The formation of the metalsilicon compound is carried out by thermally activating the diffusion of the metal into the silicon. Usually this process is done in a conventional furnace in a controlled atmosphere of $\mathrm{N}_{2}-\mathrm{H}_{2}{ }^{11}$ The process time using this technique to convert the metal layer into silicide is of the order of several minutes depending on the formation temperature. This process has been improved by the rapid thermal annealing (RTA) technique. The silicide formed by RTA is significantly better than that formed by conventional annealing, due to the 
shorter processing time ${ }^{12}$ and reduced contamination. ${ }^{10}$ More recently, new techniques have been developed based on the principle of local heating of the interface between the metal and the silicon, rather than entire substrate heating. ${ }^{13}$ The thermal energy is due to energy transfer via the absorption of an EB at the surface. With this technique, an unfocused EB is used to anneal a previously patterned metal layer deposited on the substrate surface. The present work describes a different approach to directly pattern a metal layer deposited on a substrate.

Briefly, the present method produces an etch resistant structure from a metal/semiconductor interface. The processing steps include the deposition of a metal layer capable of reacting with the semiconductor, thermally activating the diffusion of the metal into the semiconductor with a focused EB, and producing the pattern of etch-resistant metal/ semiconductor compound by displacing the beam on the surface. This is followed by wet etching the undiffused layer of metal to leave only the etch-resistant metal/semiconductor compound on the semiconductor substrate.

More specifically, this work will study the formation of $\mathrm{Pt}$ and $\mathrm{Ni}$ alloys formed on a silicon substrate or a deposited polycrystalline Si layer. The formation of Pt silicide starts with the appearance of the $\mathrm{Pt}_{2} \mathrm{Si}$ compound in the temperature range of $200-350{ }^{\circ} \mathrm{C} .{ }^{14}$ Once all the $\mathrm{Pt}$ is converted into $\mathrm{Pt}_{2} \mathrm{Si}$ and the temperature reaches the range of $300-450{ }^{\circ} \mathrm{C},{ }^{14}$ the growth of PtSi is initiated. In the case of nickel, the intermediate compounds are, in order, $\mathrm{Ni}_{2} \mathrm{Si}$, $\mathrm{NiSi}$, and $\mathrm{NiSi}_{2}$. The corresponding annealing temperatures are 200,400 , and $800{ }^{\circ} \mathrm{C} .{ }^{15}$ In a previous article, the possibility of forming $\mathrm{Pt}_{2} \mathrm{Si}$ silicide layers using a standard scanning electron microscope (SEM) has been demonstrated using Auger electron spectroscopy characterization. ${ }^{6}$

In the present work we used a JEOL JSM-6300 SEM equipped with a standard tungsten hairpin filament and a beam blanker system. The EB is controlled using the NPGS ${ }^{16}$ lithography software, which performs point by point exposures with blanking during beam displacement. The important parameters are thus beam current, point separation, and exposure time for each point. To perform the heating of the thin metal layer, a small beam energy is usually used to insure that the maximum heating occurs near the metal/ semiconductor interface. An important problem associated with using a tungsten filament is the large electron probe size at low accelerating voltages. This large probe size limits the achievable temperature underneath the EB because the current density is not very large. Typically the probe diameter for a $1 \mathrm{keV}, 100 \mathrm{pA} \mathrm{EB}$ is $\sim 360 \mathrm{~nm}$, which limits the attainable resolution. This lithography process could be enhanced using $\mathrm{a} \mathrm{LaB}_{6}$ or a field emission electron source, where typically the current density is increased by factors of 10 and 1000 , respectively.

\section{EXPERIMENTS}

The objective of the present work is to establish the framework for the application of this method to the fabrication of more complex devices such as MESFET or
MOSFET. Also, electrical characterization will be performed, to measure the conductivity of the silicide and the quality of the silicide/silicon interface.

Several types of samples were fabricated to fulfill these objectives. The first type (type I) were intended to form a Schottky contact between the silicide and the silicon. A platinum layer of $20 \mathrm{~nm}$ in thickness was evaporated on clean $n$-type high resistivity silicon $\langle 100\rangle$ substrates using an electron gun deposition system with an evaporation rate of 0.5 $\mathrm{nm} / \mathrm{s}$. Prior to this evaporation, $\mathrm{P}^{+}$ions implantation at 40 $\mathrm{keV}$ was performed on the back of the samples, and annealed in a standard furnace at $950{ }^{\circ} \mathrm{C}$ in preparation for ohmic contacts.

The second type (type II) of samples were fabricated to investigate the resistivity of the silicide and also the possibility of producing MOS capacitances. A 100-nm-thick oxide layer was first grown on a clean $\langle 100\rangle p$-type silicon substrate in a furnace at $1050^{\circ} \mathrm{C}$. A $20 \mathrm{~nm}$ layer of polycrystalline silicon is then deposited using low-pressure chemical vapor deposition (LPCVD) at $620^{\circ} \mathrm{C}$. The final step is to evaporate a thin layer of $\mathrm{Pt}$ or $\mathrm{Ni}$ using the same conditions as described previously.

Type I platinum samples were used to investigate the effect of the EB accelerating voltage. To perform this test, narrow lines of $20 \mu \mathrm{m}$ in length were exposed with various line doses. The distance between each exposed point of the line was fixed at $3.4 \mathrm{~nm}$ and the beam current was set at 100 pA in this test, with only the point exposure time as a variable. The effect of repeated exposure was also investigated. This technique means that a single line is exposed more than one time. Area doses were studied using $2 \mu \mathrm{m} \times 2 \mu \mathrm{m}$ squares. The effect of multiple exposure was once again examined as well as the variation of the distance between exposure points, in both cases with a fixed probe current. Using the same pattern, the difference in threshold doses for Pt and $\mathrm{Ni}$ silicide was determined. The threshold dose for an area is defined as the minimum dose to produce a complete defectless square. For single lines the threshold dose is determined by the minimum dose required to form a continuous line. In the case of doses smaller than this threshold value, the lines include gaps.

In order to characterize the silicide/silicon junction, a 100 $\mu \mathrm{m} \times 50 \mu \mathrm{m}$ bonding pad was exposed with a dose of 240 $\mathrm{mC} / \mathrm{cm}^{2}$ on Pt using a type I sample. The ohmic contacts were prepared using an $\mathrm{Au}-\mathrm{Sb}$ eutectic alloy.

The final pattern used in this study is intended to measure the electrical resistivity of silicide structures produced with this lithography process. It consists of three parallel silicide lines connected to bonding pads. Geometrical considerations were used to calculate the resistivity of the structures.

After EB exposure, the unreacted metal layer is removed by wet chemical etching in an aqua region solution. The ratio of the acid constituents depends on the type of sample being etched. Table I presents a summary of the various recipes used in these experiments. Following the wet chemical etching step, a thin layer of metal rich silicon remains on the surface of the samples. This is due to the formation, at room 
TABLE I. Etch parameters for different types of samples.

\begin{tabular}{ccc}
\hline \hline Sample type & $\mathrm{H}_{2} \mathrm{O}-\mathrm{HCl}-\mathrm{HNO}_{3}$ & $\begin{array}{c}\text { Etch time }\left(\text { at } 80{ }^{\circ} \mathrm{C}\right) \\
\text { (min) }\end{array}$ \\
\hline Pt on $\mathrm{Si}$ & $8: 7: 1$ & 4 \\
$\mathrm{Pt}$ on poly-Si & $22: 7: 1$ & 7 \\
$\mathrm{Ni}$ on $\mathrm{Si}$ & $42: 7: 1$ & 1 \\
$\mathrm{Ni}$ on poly-Si & $42: 7: 1$ & 1 \\
\hline \hline
\end{tabular}

temperature, of a very thin $(\approx 2 \mathrm{~nm})$ silicide layer following metal evaporation at the metal-silicon interface. ${ }^{17}$ In order to remove this layer and restore the initial surface conditions provided by the silicon substrate, type I samples are processed using a 3 min plasma etch in a $\mathrm{CF}_{4}-\mathrm{O}_{2}$ gas mixture. Type II samples are also processed in the same fashion, but the plasma etch now removes the polycrystalline silicon layer outside of the silicide structures.

In the case of electrical measurements, reference samples were prepared using evaporation masks followed by conventional RTA.

\section{RESULTS AND DISCUSSION}

\section{A. Exposure study}

Figure 1 shows the threshold line dose versus the number of repeated single line exposures. The experimental parameters are as follows: 1 and $3 \mathrm{keV} \mathrm{EB}, 100 \mathrm{pA}$ current and a $20 \mathrm{~nm}$ Pt thin film on silicon. We obtain the threshold line dose from the minimum total exposure required to form a continuous silicide line after etching. In order to reduce the carbon contamination and the local charging effects, the complete dose was achieved by multiple line exposure. The effect of carbon contamination is to absorb some of the electron energy, thus reducing the amount of energy lost in the metal layer and decreasing the temperature rise. This technique of multiple exposure reduces the total line dose required for reaching, threshold by reducing the rate of carbon deposition, ${ }^{3}$ due to the shorter exposure time of each point. From Fig. 1, it is seen that the total minimum dose is $\sim 1.2$ $\mu \mathrm{C} / \mathrm{cm}$ and is obtained with more than seven exposures.

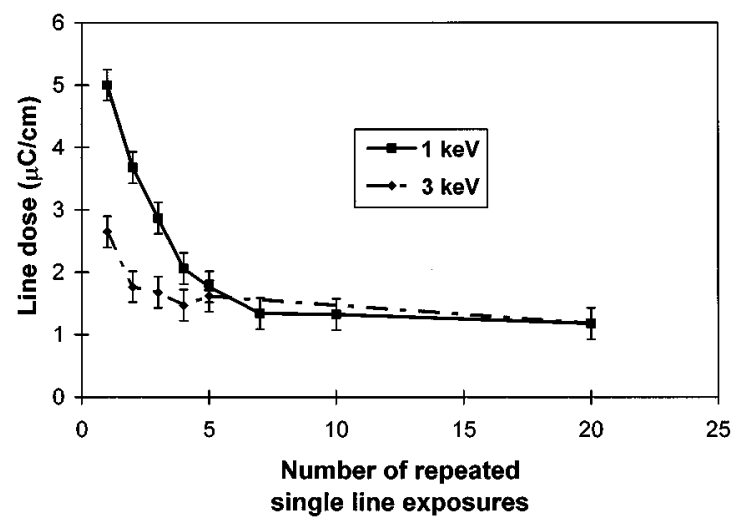

FIG. 1. Threshold, line dose as a function of the number of repeated single line exposures at 1 and $3 \mathrm{keV}$.

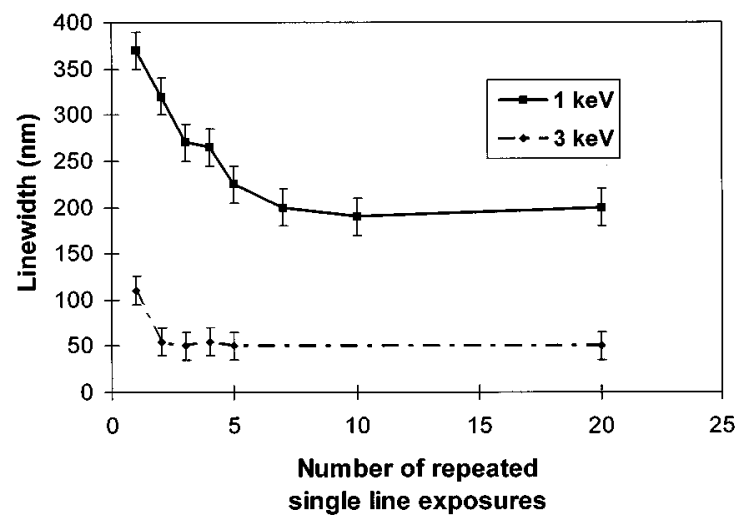

FIG. 2. Measured linewidth at threshold exposure dose as a function of the number of repeated single line exposures at 1 and $3 \mathrm{keV}$.

When using a $3 \mathrm{keV}$ EB under the same conditions, the undesirable effect of carbon contamination should be reduced. Carbon deposition is still present, but at $3 \mathrm{keV}$ the energy loss by the primary electrons in the carbon layer will be less significant due to the greater penetration depth of the electrons. This implies a reduction in the number of multiple exposures required to obtain the threshold dose as seen in Fig. 1, where the minimum dose is obtained after four exposures. Repeated line exposure also significantly decreases the linewidth at threshold, as shown in Fig. 2. For a given line dose, multiple exposure implies a series of shorter exposure times for the pattern. In this case, the temperature required for silicide formation may only be attained in the center of the beam due to its Gaussian profile, thus creating the silicide in a region which is narrower than the beam diameter. It is important to note that the effect of overetching the samples does not affect the measured linewidths. Increasing the EB energy, also significantly reduces the beam diameter and thus produces finer structure. ${ }^{18}$ In fact, for a similar line dose at 1 and $3 \mathrm{keV}$, the line width at $3 \mathrm{keV}$ is decreased by a factor of 5 .

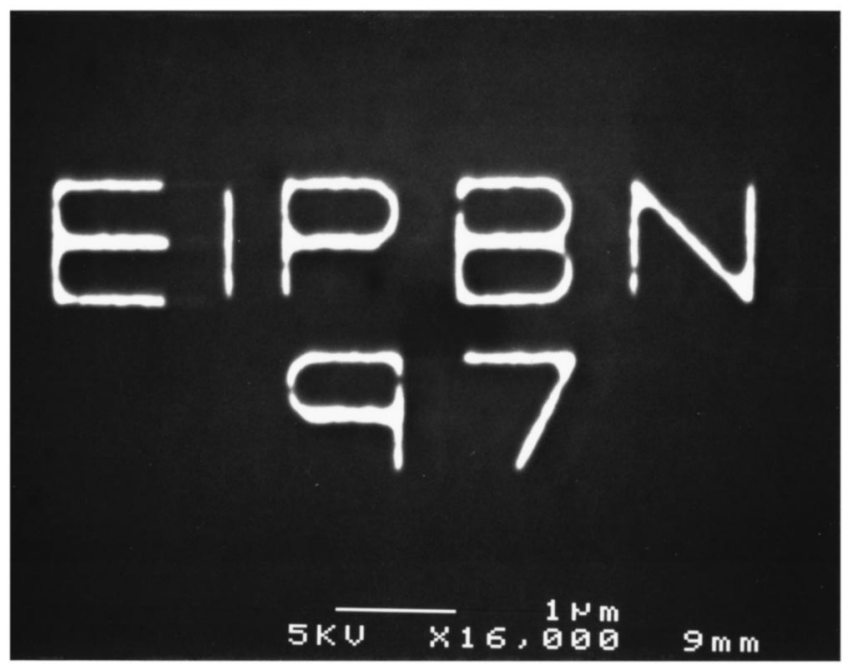

FIG. 3. Micrograph of silicide nanostructure. 
TABLE II. Effect of exposure point separation on area threshold dose.

\begin{tabular}{cc}
\hline \hline $\begin{array}{c}\text { Point separation } \\
(\mathrm{nm})\end{array}$ & $\begin{array}{c}\text { Threshold dose } \\
\left(\mathrm{mC} / \mathrm{cm}^{2}\right)\end{array}$ \\
\hline 3.4 & 65 \\
7.0 & 40 \\
14.0 & 46 \\
\hline \hline
\end{tabular}

As an example, Fig. 3 shows a platinum silicide nanostructure fabricated with this technique. The letters are $\sim 1$ $\mu \mathrm{m}$ in height and some lines have widths $<50 \mathrm{~nm}$. The experimental conditions used in this case are: $100 \mathrm{pA} \mathrm{EB}$ $\left(\approx 200 \mathrm{~mA} / \mathrm{cm}^{2}\right), 1.5 \mu \mathrm{C} / \mathrm{cm}$ dose and $3 \mathrm{keV}$ accelerating voltage. Under these conditions, the beam diameter is $\sim 250$ $\mathrm{nm}$. It is thus interesting to observe that within this figure, proximity effects are minimal and occur only at the junction of lines.

For area doses, the square pattern is exposed through a series of closely spaced horizontal lines. The distance between each exposed line in this case is much less than the beam diameter. Thus, the effect of repeated exposures is not significant since this situation is always equivalent to a high number of repeated single line exposures.

Table II summarizes the results obtained by studying the effect of exposure point separation used in the square patterns for Pt samples. Normally, no effect should be observed because the area dose is independent of the point separation. The results of Table II shows some variation, but this is within the experimental errors.

A second metal, nickel, has also been studied. Since the previously studied effects should not be influenced by the type of metal, only the threshold doses should vary. Indeed, this parameter was measured to be four times greater than for the case of $\mathrm{Pt}$ thin film. This is related to a weaker resistance of nickel silicide to the chemical etching and different silicide formation temperature.

\section{B. Electrical characterization}

Figure 4 shows the forward and reverse current-voltage

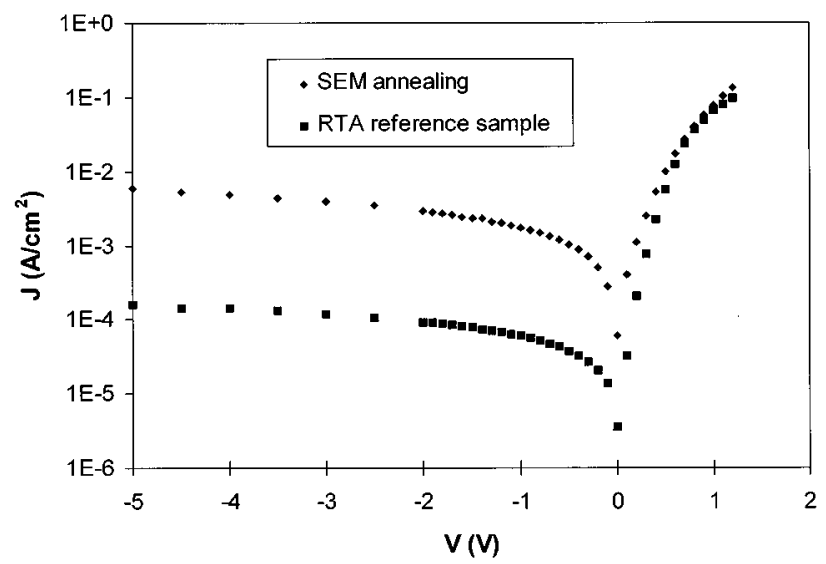

FIG. 4. Forward and reverse current-voltage relationship for a $\mathrm{Pt} / \mathrm{Si}$ interface annealed with the SEM and by RTA.

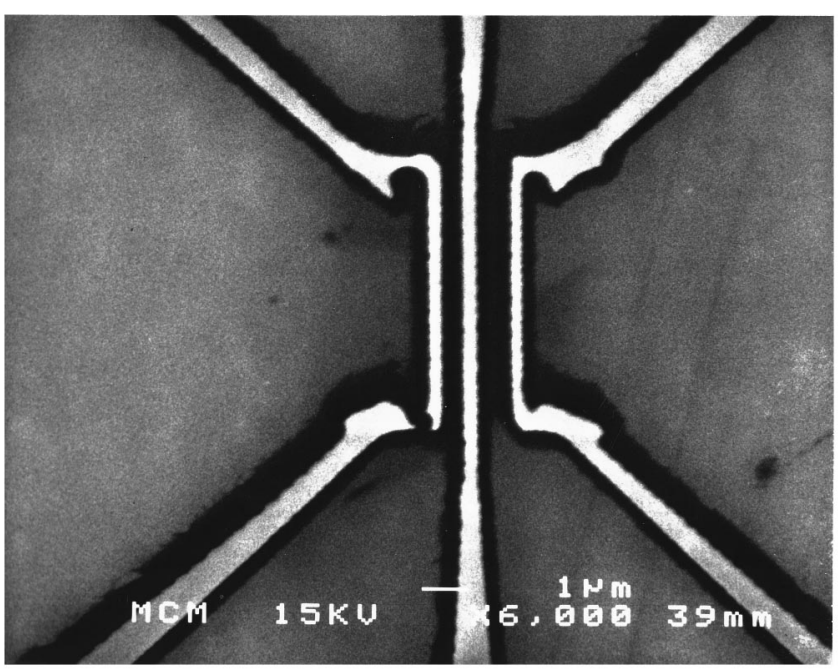

FIG. 5. Micrograph of silicide nanostructure used for electrical measurements.

relationship for a Pt/Si interface annealed with the SEM and by RTA. From the reverse saturation current the barrier height $\left(\phi_{b}\right)$ can be determined for both cases. These are $\phi_{b}$ $(\mathrm{SEM})=0.56 \mathrm{eV}$ and $\phi_{b}(\mathrm{RTA})=0.65 \mathrm{eV}$. The theoretical value of $\phi_{b}$ for $\mathrm{Pt}$ silicide on high resistivity silicon is on the order of $0.55 \mathrm{eV}$. Dimitriadis ${ }^{19}$ has shown that the method (RTA or conventional furnace) and temperature of the anneal process has a strong influence on the obtained barrier height. In our experiments, the temperature used to anneal the samples is likely to be different by RTA and SEM annealing. While it is difficult to evaluate precisely the impact of the differences between the two techniques, these barrier height values are well within the range of values observed by Dimitriadis. $^{19}$

In order to characterize a MOS structure, a similar contact pad used for Schottky measurements was exposed on a type II sample with a Pt layer to produce a MOS capacitance. In this case, the back ohmic contact was fabricated using aluminum thin film deposition. Electrical measurement of the capacitance under zero bias revealed that the pad formed on the undoped polycrystalline silicon was indeed conductive, and a value of $20 \mathrm{pF}$ was obtained.

The resistivity of the silicide produced with this method was determined using the structure illustrated in Fig. 5. This design was intended for multiple purposes: testing the fabrication of a structure containing closely spaced lines, verifying the interline electrical isolation and measuring the resistivity of the silicide structures. As shown in the example of Fig. 5, the line spacing is $<0.4 \mu \mathrm{m}$ with linewidths of the same order. In addition to confirming the electrical isolation between the lines, the resistivity of the silicide was determined to be $\sim 45 \mu \Omega \mathrm{cm}$. This value is reasonable since it is expected to be greater than the resistivity of pure platinum which is $10.6 \mu \Omega \mathrm{cm}$. 


\section{SUMMARY}

In this work, we have used a novel technique using a focused EB to generate silicide structures on a silicon substrate and a polycrystalline silicon thin film. Threshold, doses for lines and square areas have been obtained in order to establish a framework for the fabrication of more complex devices. While the resolution limit of this process has not been established, linewidths $<50 \mathrm{~nm}$ have been obtained. Preliminary electrical measurements have been carried out on silicide structures produced with this technique. This indicates that this method could be used in the fabrication of MES and MOS devices.

\section{ACKNOWLEDGMENTS}

Part of this research was funded by Micronet and the Natural Sciences and Engineering Research Council of Canada (NSERC). One of the authors (D.D.) would like to acknowledge support from NSERC. They would also like to thank Jacques Corbin and Pierre Lafrance for technical support.

${ }^{1}$ D. Wang, P. C. Hoyle, J. R. A. Cleaver, G. A. Porkolab, and N. C. MacDonald, J. Vac. Sci. Technol. B 13, 1984 (1995).

${ }^{2}$ H. Sugimura and N. Nakagiri, J. Vac. Sci. Technol. B 13, 1933 (1995).
${ }^{3}$ J. Fujita, H. Watanabe, Y. Ochiai, S. Manako, J. S. Tsai, and S. Matsui, Appl. Phys. Lett. 66, 3065 (1995).

${ }^{4}$ W. Langheinrich and H. Beneking, Jpn. J. Appl. Phys., Part 1 32, 6248 (1993).

${ }^{5}$ H. Sugimura and N. Nakagiri, J. Vac. Sci. Technol. B 13, 1933 (1995).

${ }^{6}$ D. Drouin, J. Beauvais, R. Lemire, E. Lavallée, R. Gauvin, and M. Caron, Appl. Phys. Lett. 70, 3020 (1997).

${ }^{7}$ W. E. Beadle, J. C. C. Tsai, and R. D. Plummer, Quick Reference Manual for Silicon Integrated Circuit Technology (Wiley, New York, 1985).

${ }^{8}$ B.-Y. Tsaur, J. P. Mattia, and C. K. Chen, Appl. Phys. Lett. 57, 1111 (1990).

${ }^{9}$ H. Bender, P. Roussel, S. Kolodinski, A. Torres, R. A. Donaton, K. Maex, and P. Vander Sluis, Mater. Res. Soc. Symp. Proc. 402, 449 (1996).

${ }^{10}$ C. A. Dimitrias, E. K. Polychroniadis, E. K. Evangelou, and G. E. Giakoumakis, J. Appl. Phys. 70, 3109 (1991).

${ }^{11}$ B.-Y. Tsui and M.-C. Chen, J. Appl. Phys. 68, 6246 (1990).

${ }^{12}$ A. Torres, S. Kolodinski, R. A. Donaton, K. Roussel, and H. Bender, Proc. SPIE 2554, 185 (1995).

${ }^{13}$ E. D'Anna, G. Leggieri, and A. Luches, Thin Solid Films 129, 93 (1985).

${ }^{14}$ E. G. Colgan, J. Mater. Res. 10, 1953 (1995).

${ }^{15}$ S. M. Sze, Physics of Semiconductor Devices, 2nd ed. (Wiley, New York, 1992), p. 292.

${ }^{16}$ Nanometer Pattern Generation System ver. 7.5, by J. C. Nabity Lithography Systems.

${ }^{17}$ L. Ley, Y. Wang, V. Nguyen Van, S. Fisson, D. Souche, G. Vuye, and J. Rivory, Thin Solid Films 270, 561 (1995).

${ }^{18}$ P. Baumgartier, Appl. Phys. Lett. 64, 592 (1994).

${ }^{19}$ C. A. Dimitriadis, Appl. Phys. Lett. 56, 143 (1990). 